hep-th/9806131

IASSNS-HEP-98/54

BONN-TH-98-12

\title{
Spectra of 4D, N=1 Type I String Vacua on Non-Toroidal CY Threefolds
}

\author{
Ralph Blumenhagen ${ }^{1}$ and Andreas Wißkirchen ${ }^{2}$ \\ 1 School of Natural Sciences, Institute for Advanced Study, \\ Olden Lane, Princeton NJ 08540, USA \\ 2 Physikalisches Institut der Universität Bonn, \\ Nußallee 12, 53115 Bonn, Germany
}

\begin{abstract}
We compute the massless spectra of some four dimensional, $N=1$ supersymmetric compactifications of the type I string. The backgrounds are non-toroidal Calabi-Yau manifolds described at special points in moduli space by Gepner models. Surprisingly, the abstract conformal field theory computation reveals Chan-Paton gauge groups as big as $S O(12) \otimes S O(20)$ or $S O(8)^{4} \otimes S O(4)^{3}$.
\end{abstract}

$06 / 98$

${ }^{1}$ e-mail: blumenha@sns.ias.edu

2 e-mail: wisskirc@avzw02.physik.uni-bonn.de 


\section{Introduction}

Of all five ten dimensional string theories the type I branch has certainly received less attention during the perturbative decade in the history of this field. However, mainly during the last two years we have seen some extended activity in computing and better understanding type I vacua in six and four space time dimensions [1 [4]. Technically, this progress was fed by our better understanding of orientifolds and D-branes. Moreover, we know that all five string theories are related to each other by dualities and are believed to have a common ancestor in eleven dimensions, so called M-theory. From this point of view every string theory can teach us something about M-theory.

From the low energy effective actions of the type I and the $S O(32)$ heterotic string, one conjectures a strong-weak coupling duality between these two theories [5]. This duality has passed several test, even after compactification. In particular, in four dimensions one expects a purely perturbative duality between these two string theories in special regions of the moduli space. For some orientifolds there also exists a duality to a certain kind of F-theory vacua, which can be used to gain some non-perturbative insight into the former models.

So far all type I model building was restricted to toroidal orbifolds in general including 9-branes and 5-branes for tadpole cancellation. As argued in [6] consistency of such orientifolds severely constraints the number of such compactifications. For instance, absence of additional non-perturbative states and world sheet consistency allows only six possible abelian orbifold of $T^{6}$.

This paper deals with the construction of four dimensional type I vacua for background manifolds which are not toroidal orbifolds. Since the exact conformal field theory for every point in moduli space is not known, the very illustrative method of [2] can not be used. However, for some Calabi-Yau threefolds Gepner has provided exactly solvable points in terms of tensor products of $N=2$ superconformal field theories [7]. Furthermore, in a series of papers [8], purely algebraic methods to construct type I descendants of such abstract conformal field theories were developed ${ }^{1}$. The open string sector of Gepner models was also discussed in [9].

After reviewing the general construction of type I vacua in terms of rational conformal field theories in section two, in section three we apply this to the case of Gepner models

1 We would like to emphasize that already in 1991 what is now called the Gimon-Polchinski model was discussed in a paper by Bianchi and Sagnotti [1]. 
in four dimensions. From a naive approach such models are hard to come by, for their huge number of representations of the chiral algebra. We make use of simple current techniques to first transform the model to accessible size. In particular we consider a $\mathbb{Z}_{5}$ orbifold of the quintic and a $\mathbb{Z}_{3}$ orbifold of $\mathbb{P}_{1,1,1,3,3}[9]$. The type I models turn out to have interesting spectra with gauge groups like for instance $S O(12) \times S O(20)$. Unfortunately, in this formalism there is no inherent distinction between Chan-Paton (CP) factors arising from 9-branes and 5-branes, so that in contrast to toroidal orbifolds it is more involved to find dual heterotic partners. Since the threefolds we consider do not admit an elliptic fibration, there does not exist an F-theory dual model, either.

\section{Type I descendants of Type II B vacua}

For constructing vacua of closed string theories, one loop modular invariance served as the main constraint in ruling out non consistent compactifications. Fortunately, for open string vacua, i.e. for conformal field theories (CFTs) with boundaries, one loop tadpole cancellation turned out to be as restrictive. In this section we briefly review the essential steps for constructing all four type I one loop amplitudes in terms of a rational conformal field theory. For further details we refer the reader to the extensive literature [8].

One starts with a left right symmetric type IIB model with a set of representations of the maximal chiral algebra $\mathcal{A}$ with characters $\chi_{i}$. These $\chi_{i}$ transform under the two generating modular transformations with some matrices $S$ and $T$. The charge conjugation matrix is defined as $C=S^{2}$. The modular invariant torus partition function can be written as

$$
Z_{T}(\tau, \bar{\tau})=\sum_{i, j=1}^{N} \chi_{i}(\tau) N_{i j} \bar{\chi}_{j}(\bar{\tau}) .
$$

Since one started with a left-right symmetric CFT, the world sheet parity operation $\Omega$ is really a symmetry of the two dimensional world sheet theory and can thus be divided out ${ }^{2}$. As usual, modding out $\Omega$ introduces unoriented world sheets like $\mathbb{R P}_{2}$ at tree level or the Klein bottle at one loop, respectively.

2 Note, that for toroidal orbifolds, requiring that $\Omega$ is really a symmetry of the theory yields further severe conditions arising from the twisted sector two form flux [6]. Thus, the world sheet consistency conditions discussed in [6] seem automatically to be satisfied in the rational CFT approach. Whether this excludes any subtleties related to the appearance of additional non-perturbative states is not absolutely clear to us. 
In the direct (open channel) Klein bottle amplitude only left-right symmetric states are allowed to contribute, leading to

$$
K(y)=\sum_{j=1}^{N} K_{j} \chi_{j}(2 i y)
$$

where the variable $y$ is real. The coefficients have to satisfy $\left|K_{j}\right|=N_{j j}$ and the choice of signs has to be consistent with the fusion rules. In the course of this paper, we will always choose the standard Klein bottle projection with all signs positive, implying (anti)symmetrization in the (R-R) NS-NS closed string sector. The transverse (closed channel) amplitude is obtained by applying the modular S-matrix to the direct Klein bottle amplitude. Since roughly speaking the Klein bottle can be written as a cylinder with two crosscaps and the closed channel describes a closed string propagating between these two boundaries, the transverse amplitude can be written as

$$
\widetilde{K}(t)=\sum_{j=1}^{N} \Gamma_{j}^{2} \chi_{j}(i t / 2) .
$$

Integrating (2.3) over $t$, in general leads to infinities interpreted as massless tadpoles, which have to be cancelled by other contributions arising from open string diagrams.

The open channel cylinder amplitude can be written as

$$
A(y)=\sum_{j=1}^{N} \sum_{a, b} A_{a b}^{j} n^{a} n^{b} \chi_{j}(i y / 2)
$$

where the $n^{a}$ denote Chan-Paton factors and the coefficient $A_{a b}^{i}$ are non-negative integers. Since the transverse amplitude describes the propagation of a closed string between two boundaries (D-branes), it can again be written as a square

$$
\widetilde{A}(t)=\sum_{j=1}^{N}\left(\sum_{a} B_{a}^{j} n^{a}\right)^{2} \chi_{j}(2 i t) .
$$

For trivial choice of the gluing automorphism a state in $\chi_{j}$ is reflected into its charge conjugate at the boundary. Thus, only states which couple to its conjugate in $Z_{T}$ are allowed to contribute in the closed string channel (2.5). As Cardy has shown, choosing the torus partition function to be the charge conjugation one and taking the same number of 
CP factors than characters, a natural solution is $A_{i j}^{k}=N_{i j}^{k}$ [10. Moreover, in the closed string channel one obtains

$$
B_{i}^{j}=\frac{S_{i j}}{\sqrt{S_{1 j}}} .
$$

Finally, the Möbius strip also contributes at one loop in the open string sector

$$
M(y)=\sum_{j} M_{a}^{j} n^{a} \hat{\chi}_{j}\left(\frac{i y+1}{2}\right),
$$

where it is convenient to introduce the real characters

$$
\hat{\chi}\left(\frac{i y+1}{2}\right)=e^{-i \pi\left(h-\frac{c}{24}\right)} \chi\left(\frac{i y+1}{2}\right) .
$$

The transverse Möbius strip amplitude can be written as

$$
\widetilde{M}(t)=\sum_{j} \Gamma_{j}\left[\sum_{a} B_{a}^{j} n^{a}\right] \hat{\chi}_{j}\left(\frac{i t+1}{2}\right)
$$

reflecting the fact that the Möbius strip can be regarded as a cylinder with a crosscap on one side. The transverse amplitude is related to the direct amplitude (2.7) by an action of the matrix $P=T^{\frac{1}{2}} S T^{2} S T^{\frac{1}{2}}$. In order to have a consistent projection in the open sector the cylinder and Möbius strip amplitude must satisfy

$$
M_{a}^{j}=A_{a a}^{j} \bmod 2
$$

As was shown in [8], besides the fusion rule coefficients one can define another integer valued object [1]

$$
Y_{i j}^{k}=\sum_{l} \frac{S_{i l} P_{j l} P_{k l}^{*}}{S_{1 l}} .
$$

For the charge conjugation modular invariant, $Y_{i j}^{k}$ determines a consistent $\mathrm{CP}$ assignment by

$$
K_{j}=Y_{j 1}^{1}, \quad M_{i}^{j}=Y_{i j}^{1}
$$

In the following we will only make use of this choice of CP factors. Summarizing, in order to compute type I one loop amplitudes one has to specify a rational CFT including a set of characters, yielding a representation of the modular group, and the matrices $S, T$ and $P$. 
Suppose, a second modular invariant torus amplitude $Z_{T}^{\prime}$ is related to the charge conjugation one $Z_{T}^{c}$ by a simple current acting without fixed points. Thus, every orbit has the same length $L$. Denote by $M^{\prime}$ the set of those $\chi_{i}$ coupling to its charge dual in $Z_{T}^{\prime}$. Then, one can easily show that the two Klein bottle amplitudes are related by

$$
\widetilde{K}_{j}^{\prime}= \begin{cases}L \widetilde{K}_{j}^{c} & \text { for } j \in M^{\prime} \\ 0 & \text { for } j \notin M^{\prime}\end{cases}
$$

If one identifies $\mathrm{CP}$ factors lying in the same orbit under the simple current one can naturally define a new cylinder and Möbius strip amplitude

$$
\begin{aligned}
A^{\prime} & =\left.\frac{1}{L} A^{c}\right|_{\text {identify } C P s}, \\
M^{\prime} & =\left.M^{c}\right|_{\text {identify } C P s}
\end{aligned}
$$

automatically satisfying all the consistency requirements and $A_{j}^{\prime}=0$ for $j \notin M^{\prime}$. The generalization of this procedure to simple currents with fixed points was discussed in [8, 12]. For all cases studied in the sequel of this paper, the two formulae (2.13) and (2.14) are however sufficient.

In the case of a four dimensional Gepner model with $N_{f}$ tensor factors the characters $\chi_{i}$ are orbits under a set of simple current including the GSO projection ${ }^{3}$

$$
J=\left(\Phi_{1,1}^{0}\right)^{N_{f}} \otimes s_{S O(2)},
$$

where $s_{S O(2)}$ denotes the spinor representation of $S O(2)$ arising from two transverse fermions which are superpartners of the two bosonic space time coordinates $X^{\mu}$. Remember, as q-series these orbits vanish due to supersymmetry. The overall integrated transverse Klein bottle amplitude looks like

$$
\widetilde{K}=\int_{0}^{\infty} d z \frac{4}{\eta^{2}(i z)} \sum_{j=1}^{N} \Gamma_{j}^{2} \chi_{j}(i z) .
$$

Similarly one obtains for the cylinder amplitude

$$
\widetilde{A}=\int_{0}^{\infty} d z \frac{1}{4 \eta^{2}(i z)} \sum_{j=1}^{N}\left[\sum_{a} B_{a}^{j} n^{a}\right]^{2} \chi_{j}(i z)
$$

3 We denote the primary field with $N=2$ quantum numbers $(l, m, s)$ by $\Phi_{m, s}^{l}$. 
and for the Möbius amplitude

$$
\widetilde{M}=\int_{0}^{\infty} d z \frac{2}{\hat{\eta}^{2}\left(\frac{1}{2}+i z\right)} \sum_{j} \Gamma_{j}\left[\sum_{a} B_{a}^{j} n^{a}\right] \hat{\chi}_{j}\left(\frac{1}{2}+i z\right) .
$$

Tadpole cancellation means that the overall coefficients of the massless orbits $M$ in the sum of these three terms $\widetilde{K}+\widetilde{A}+\widetilde{M}$ must vanish. This usually yields a highly non-trivial set of linear equations for the positive integer valued CP factors. In contrast to the advanced model building machinery in heterotic or type II string theory, type I models really need a thorough case by case study. After determining the CP factors, the closed and open string spectrum is determined by the projections $\frac{1}{2}\left(Z_{T}+K\right)$ and $\frac{1}{2}(A+M)$, respectively.

\section{Type I on $T^{6} / \mathbb{Z}_{3}$}

In reference [13] Gepner models for six dimensional vacua were considered, where special emphasis was put in models corresponding to toroidal orbifolds decribed by free $N=2$ super conformal field theories. The main problem one faces in extending such an analysis to four dimensions, is the huge number of spectral flow invariant orbits $\chi_{i}$, which is of order $10^{3}$. One way to reduce this number to smaller size (order $10^{2}$ ) is to first divide by a further simple current of integer conformal dimension and order $K$. This introduces new projections leading to a theory with order $10^{3} / K^{2}$ independent characters. In general a simple current might have fixed points, which means orbits of shorter length, so that one first has to resolve these fix points in order to define a bona fide conformal field theory [14]. For instance, if one of the levels of the $N=2$ tensor factors is even, fix point resolution becomes already inevitable. In the following some of the very few cases without fix points are considered.

As a first warm off example we consider the Gepner model $(1)^{9}$ containing 2187 orbits of integer charge [13]. Further projection with the two integer dimensional simple currents

$$
\begin{aligned}
& J_{1}=\Phi_{0,0}^{0} \otimes \Phi_{0,0}^{0} \otimes \Phi_{0,0}^{0} \otimes \Phi_{-1,0}^{1} \otimes \Phi_{-1,0}^{1} \otimes \Phi_{-1,0}^{1} \otimes \Phi_{1,0}^{1} \otimes \Phi_{1,0}^{1} \otimes \Phi_{1,0}^{1} \\
& J_{2}=\Phi_{0,0}^{0} \otimes \Phi_{-1,0}^{1} \otimes \Phi_{1,0}^{1} \otimes \Phi_{0,0}^{0} \otimes \Phi_{-1,0}^{1} \otimes \Phi_{1,0}^{1} \otimes \Phi_{0,0}^{0} \otimes \Phi_{-1,0}^{1} \otimes \Phi_{1,0}^{1}
\end{aligned}
$$

reduces the number of orbits to 27 . The charge conjugation modular invariant leads to a type II B model with 36 hypermultiplets and no vectormultiplet. This is the same spectrum as for the $\mathbb{Z}_{3}$ orbifold of the six torus $T^{6}$ with Hodge numbers $h_{11}=36$ and $h_{21}=0$. It was shown in [4] that the geometric type I orientifold has maximal gauge group $U(12) \times S O(8)$. 
We will see that in the Gepner model framework we do not come even close. Both the charge conjugation and the diagonal torus partition function yield type I descendants with 36 neutral chiral multiplets in the closed string sector and with maximal gauge group $S p(4)$ in the open string sector. For the diagonal invariant this spectrum is enlarged by 36 further chiral multiplets in the adjoint of $S p(4)$. The result for the charge conjugation invariant is consistent with the prediction that a backgound two form field of rank $r=6$ reduces the rank of the gauge group by a factor $2^{r / 2}$ [6,15]. Thus, as this model seems to suggest, Gepner models might lead only to type I descendants which lie on some fairly uninteresting branches of the moduli space. Nevertheless, for non-toroidal Calabi-Yau manifolds we do not have any better technique available and we will see in the next section that more interesting gauge groups can arise.

\section{Type I on an orbifold of the Quintic}

Consider the type II B compactification on the quintic $\mathbb{P}_{4}[5]$. At the Landau-Ginzburg point in Kähler moduli space it is decribed by the conformal field with tensor product $(k=3)^{5}$ in the internal sector. By taking orbits with respect to the GSO projections one obtains $n=4000$ characters, 202 of them are massless and for the diagonal invariant give rise to the well known spectrum of $h_{21}=101$ vector multiplets and $h_{11}=1$ hypermultiplets. We use the freely acting simple current

$$
J=\Phi_{0,0}^{0} \otimes \Phi_{-3,0}^{3} \otimes \Phi_{-1,0}^{3} \otimes \Phi_{1,0}^{3} \otimes \Phi_{3,0}^{3}
$$

to reduce the number of characters to $n=160$. Now, only 42 of them are massless and the diagonal type II B spectrum consists of $h_{21}=21$ vector multiplets and $h_{11}=1$ hypermultiplets. This is equivalent to modding out the quintic by the discrete $\mathbb{Z}_{5}$ subgroup $z_{m} \rightarrow e^{2 \pi i \frac{m}{5}} z_{m}$.

\subsection{The charge conjugation invariant}

Calculating the matrices $S, C, T$ and $P$ for these 160 characters, it turns out that there exist 32 self-conjugate orbits $O_{c}$. The massless orbits imply 42 tadpole cancellation conditions for the $160 \mathrm{CP}$ factors. These conditions are linear in the CPs, but of course have to be solved over non-negative integers. Even the fastest computer can not check all 
possibilities. One solution is obvious from the fact, that there exists an orbit $\Phi$ with fusion rules

$$
\Phi \times \Phi=\sum_{i \in O_{c}} \Phi_{i}
$$

Thus, there exists a CP factor $n$ such that $A=n^{2} K$ and one obtains only one independent tadpole cancellation condition, which is solved by $n=4$. The massless spectrum consists

of the usual $N=1$ supergravity multiplet, a chiral multiplet containing the dilaton, 22 further neutral chiral multiplets all appearing in the projected closed string sector. In the open sector one finds a vector multiplet in the adjoint of $S O(4)$ and in each case one chiral multiplet in the symmetric, $\mathbf{9}$, and in the singlet, $\mathbf{1}$, representation of $S O(4)$.

Similar solutions with gauge group $S O(4)$ exist in all the other examples studied in this paper, as well. Note, that the reduction of the open string gauge group to $S O(4)$ or $S p(4)$ is what one would naively expect for a toroidal orbifold with an NS two form background of maximal rank [15].

\subsection{The diagonal invariant}

Instead of using the charge conjugate torus partition function we can also use the diagonal one. Then, the Klein bottle amplitude contains all 160 orbits. In order to find the CP assignment in the cylinder amplitude via (2.13) and (2.14) we have to know a simple current relating the charge conjugate invariant to the diagonal invariant. This is easy to find and is given by

$$
J=\Phi_{0,0}^{0} \otimes \Phi_{1,0}^{3} \otimes \Phi_{-1,0}^{3} \otimes \Phi_{-1,0}^{3} \otimes \Phi_{1,0}^{3}
$$

Identifying all CPs in the charge conjugate annulus amplitude lying in the same orbits under (4.3) yields 32 CP factors for the diagonal invariant. Since there are now only two(!) non-trivial tadpole cancellation conditions for 32 variables we can solve the equations in full generality, revealing a vast amount of different solutions. The largest gauge group one can get is $S O(12) \times S O(20)$ without any charged matter. The massless spectrum from the closed sector is the same as for the charge conjugation invariant. A few other solutions of the tadpole cancellation condition are presented in Table 1. Since one expects non-trivial superpotentials to arise, a more detailed investigation is needed to decide which models lie on the same moduli. 


\begin{tabular}{|c|c|c|c|}
\hline Invariant & closed sector & gauge group & matter \\
\hline$C$ & $22 \times \mathbf{1}_{L}$ & $S O(4)$ & $\begin{array}{l}\mathbf{9}_{L} \\
\mathbf{1}_{L}\end{array}$ \\
\hline$D$ & $22 \times \mathbf{1}_{L}$ & $S O(12) \times S O(20)$ & - \\
\hline$D$ & $22 \times \mathbf{1}_{L}$ & $S O(12) \times S O(20-n) \times S O(n)$ & $(\mathbf{1}, \mathbf{2 0}-\mathbf{n}, \mathbf{n})_{L}$ \\
\hline$D$ & $22 \times \mathbf{1}_{L}$ & $S O(10) \times S O(18) \times U(1)$ & $\begin{array}{l}\mathbf{1 0}, \mathbf{1} ; \pm 1)_{L} \\
(\mathbf{1}, \mathbf{1 8} ; \pm 1)_{L} \\
(\mathbf{1}, \mathbf{1} ; 0)_{L}\end{array}$ \\
\hline$D$ & $22 \times \mathbf{1}_{L}$ & $S O(6) \times S O(8) \times S O(6)$ & $\begin{array}{l}(\mathbf{6}, \mathbf{1}, \mathbf{6})_{L} \\
2 \times(\mathbf{1}, \mathbf{8}, \mathbf{6})_{L} \\
3 \times(\mathbf{1}, \mathbf{1}, \mathbf{1 5})_{L} \\
(\mathbf{1}, \mathbf{1}, \mathbf{2 0})_{L} \\
(\mathbf{1}, \mathbf{1}, \mathbf{1})_{L}\end{array}$ \\
\hline
\end{tabular}

Table 1: massless spectra for type I quintic descendant

Unfortunately, in the rational CFT setting we can not decide whether, for instance, the $S O(12) \times S O(20)$ model contains any 5-branes or is perturbative from the heterotic viewpoint. Finding a possible heterotic dual would be interesting but is beyond the scope of this paper. Note, that all models in Table 1 are non-chiral and as expected from one-loop consistency all gauge anomalies vanish. We would like to emphasize, that the geometric reason for getting so many solutions is that the number of Kähler moduli and therefore the number of tadpole cancellation conditions for this peculiar Calabi-Yau is so small, namely $h_{11}=1$. Thus, in order to see similar patterns in other Gepner models, one should in particular study cases with small numbers of Kähler moduli and interacting $N=2$ superconformal field theories.

\section{Type $I$ on an orbifold of $\mathbb{P}_{1,1,1,3,3}[9]$}

The second model we want to study is an orbifold of the Calabi-Yau $\mathbb{P}_{1,1,1,3,3}[9]$, which has Hodge numbers $h_{21}=104$ and $h_{11}=4$. The model itself contains the solvable Gepner point $\left(1^{2} 7^{3}\right)$ and gives rise to 5184 orbits after imposing the GSO projection. Reducing this number to 576 is possible by also projecting with the simple current

$$
J=\Phi_{0,0}^{0} \otimes \Phi_{0,0}^{0} \otimes \Phi_{-3,0}^{7} \otimes \Phi_{3,0}^{7} \otimes \Phi_{0,0}^{0}
$$


of order 3. The Hodge numbers of the orbifold theory are $h_{21}=46$ and $h_{11}=10$. The calculation is similar to the one for the quintic. For the charge conjugation invariant we obtain 67 tadpole cancellation conditions, two solutions of which are displayed in Table 2 . In this case, we did not find any solution with gauge groups bigger than products of $S O(4)$ factors.

\begin{tabular}{|c|c|c|c|}
\hline Invariant & closed sector & gauge group & matter \\
\hline$C$ & $56 \times \mathbf{1}_{L}$ & $S O(4)$ & $\begin{array}{l}7 \times \mathbf{9}_{L} \\
7 \times \mathbf{1}_{L} \\
1 \times \mathbf{6}_{L}\end{array}$ \\
\hline C & $56 \times \mathbf{1}_{L}$ & $S O(4)^{3}$ & $\begin{array}{l}2 \times(\mathbf{9}, \mathbf{1}, \mathbf{1})_{L} \\
2 \times(\mathbf{1}, \mathbf{1}, \mathbf{1})_{L} \\
(\mathbf{4}, \mathbf{4}, \mathbf{1})_{L} \\
(\mathbf{1}, \mathbf{4}, \mathbf{4})_{L} \\
(\mathbf{4}, \mathbf{1}, \mathbf{4})_{L}\end{array}$ \\
\hline$D$ & $56 \times \mathbf{1}_{L}$ & $S O(8)^{4} \times S O(4)^{3}$ & $\begin{array}{l}2 \times(\mathbf{8}, \mathbf{8}, \mathbf{1}, \mathbf{1} ; \mathbf{1}, \mathbf{1}, \mathbf{1})_{L} \\
2 \times(\mathbf{8}, \mathbf{1}, \mathbf{8}, \mathbf{1} ; \mathbf{1}, \mathbf{1}, \mathbf{1})_{L} \\
2 \times(\mathbf{8}, \mathbf{1}, \mathbf{1}, \mathbf{8} ; \mathbf{1}, \mathbf{1}, \mathbf{1})_{L} \\
1 \times(\mathbf{8}, \mathbf{1}, \mathbf{1}, \mathbf{1} ; \mathbf{4}, \mathbf{1}, \mathbf{1})_{L} \\
1 \times(\mathbf{8}, \mathbf{1}, \mathbf{1}, \mathbf{1} ; \mathbf{1}, \mathbf{4}, \mathbf{1})_{L} \\
1 \times(\mathbf{8}, \mathbf{1}, \mathbf{1}, \mathbf{1} ; \mathbf{1}, \mathbf{1}, \mathbf{4})_{L} \\
1 \times(\mathbf{1}, \mathbf{8}, \mathbf{8}, \mathbf{1} ; \mathbf{1}, \mathbf{1}, \mathbf{1})_{L} \\
1 \times(\mathbf{1}, \mathbf{8}, \mathbf{1}, \mathbf{8} ; \mathbf{1}, \mathbf{1}, \mathbf{1})_{L} \\
2 \times(\mathbf{1}, \mathbf{8}, \mathbf{1}, \mathbf{1} ; \mathbf{4}, \mathbf{1}, \mathbf{1})_{L} \\
1 \times(\mathbf{1}, \mathbf{8}, \mathbf{1}, \mathbf{1} ; \mathbf{1}, \mathbf{4}, \mathbf{1})_{L} \\
2 \times(\mathbf{1}, \mathbf{8}, \mathbf{1}, \mathbf{1} ; \mathbf{1}, \mathbf{1}, \mathbf{4})_{L} \\
1 \times(\mathbf{1}, \mathbf{1}, \mathbf{8}, \mathbf{8} ; \mathbf{1}, \mathbf{1}, \mathbf{1})_{L} \\
2 \times(\mathbf{1}, \mathbf{1}, \mathbf{8}, \mathbf{1} ; \mathbf{4}, \mathbf{1}, \mathbf{1})_{L} \\
2 \times(\mathbf{1}, \mathbf{1}, \mathbf{8}, \mathbf{1} ; \mathbf{1}, \mathbf{4}, \mathbf{1})_{L} \\
1 \times(\mathbf{1}, \mathbf{1}, \mathbf{8}, \mathbf{1} ; \mathbf{1}, \mathbf{1}, \mathbf{4})_{L} \\
1 \times(\mathbf{1}, \mathbf{1}, \mathbf{1}, \mathbf{8} ; \mathbf{4}, \mathbf{1}, \mathbf{1})_{L} \\
2 \times(\mathbf{1}, \mathbf{1}, \mathbf{1}, \mathbf{8} ; \mathbf{1}, \mathbf{4}, \mathbf{1})_{L} \\
2 \times(\mathbf{1}, \mathbf{1}, \mathbf{1}, \mathbf{8} ; \mathbf{1}, \mathbf{1}, \mathbf{4})_{L} \\
1 \times(\mathbf{1}, \mathbf{1}, \mathbf{1}, \mathbf{1} ; \mathbf{4}, \mathbf{4}, \mathbf{1})_{L} \\
1 \times(\mathbf{1}, \mathbf{1}, \mathbf{1}, \mathbf{1} ; \mathbf{4}, \mathbf{1}, \mathbf{4})_{L} \\
1 \times(\mathbf{1}, \mathbf{1}, \mathbf{1}, \mathbf{1} ; \mathbf{1}, \mathbf{4}, \mathbf{4})_{L}\end{array}$ \\
\hline
\end{tabular}

Table 2: massless spectra for type I descendant of $(1)^{2}(7)^{3}$ Gepner model 
The diagonal invariant leads to only nine conditions for $64 \mathrm{CP}$ factors. A lot of solutions can be found by computer search, however, there is no chance of classifying them all. In Table 2 we present the solution we found with maximal rank of the gauge group. Remarkably, the rank is 22 so that we can conclude that there must be 5-branes present in this vacuum and it must correspond to a non-perturbative vacuum on the dual heterotic side. The lesson we learn from the latter two examples is, that in contrast to some expectations Gepner models can provide four dimensional, $N=1$ supersymmetric type I backgrounds with interesting gauge groups.

\section{Summary}

In this paper we have started an investigation of four dimensional type I vacua with $N=1$ supersymmetry derived from Gepner models. Geometrically, these vacua correspond to compactifications on Calabi-Yau manifolds. We have computed only the simplest nontoroidal models, but we think the results are encouraging for further study in this rich class of models. To get interesting results, one should in particular consider models with small numbers of Kähler moduli. Technically, there exist some generalizations to the constuction presented in this paper like the choice of non-trivial Klein Bottle projections or the choice of non-trivial gluing automorphisms for the cylinder amplitude.

With respect to the question of heterotic dual models, it would be very nice to have an intrinsic condition to distinguish type I backgrounds with and without 5-branes. Furthermore, the existence of $(0,2)$ target space dualities for perturbative heterotic vacua [16] suggests that such dualities should also hold for at least a subclass of type I vacua.

\section{Acknowledgments}

It is a pleasure to thank Angel Uranga and Jaemo Park for discussion and Zurab Kakushadse for valuable e-mail correspondence. Moreover, we would like to thank Jürgen Fuchs, Christoph Schweigert and Andreas Recknagel for some comments about an earlier version of this paper. This work is supported by NSF grant PHY-9513835. 


\section{References}

[1] M. Bianchi and A. Sagnotti, On the Systematics of Open String Theories, Phys. Lett. B247 (1990) 517; Twist Symmetry and Open String Wilson Lines, Nucl. Phys. B361 (1991) 519

[2] E.G. Gimon and J. Polchinski, Consistency Conditions for Orientifolds and DManifolds, Phys. Rev. D54 (1996) 1667, hep-th/9601038

[3] E.G. Gimon and C.V. Johnson, K3 Orientifolds, Nucl. Phys. B477 (1996) 715, hepth/9604129;

A. Dabholkar and J. Park, Strings on Orientifolds, Nucl. Phys. B477 (1996) 701

[4] M. Berkooz and R.G. Leigh, A D =4 N=1 Orbifold of Type I Strings, Nucl. Phys. B483 (1997) 187, hep-th/9605049;

C. Angelantonj, M. Bianchi, G. Pradisi, A. Sagnotti and Ya.S. Stanev, Chiral Asymmetry in Four-Dimensional Open-String Vacua, Phys. Lett. B385 (1996) 96, hepth/9606169;

Z. Kakushadze, Aspects of $N=1$ Type I-Heterotic Duality in Four Dimensions, Nucl. Phys. B512 (1998) 221, hep-th/9704059;

Z. Kakushadze and G. Shiu, A Chiral $N=1$ Type I Vacuum in Four Dimensions and Its Heterotic Dual, Phys. Rev. D56 (1997) 3686, hep-th/9705163; $4 D$ Chiral $N=1$ Type I Vacua with and without D5-branes, Nucl. Phys. B520 (1998) 75, hepth/9706051;

Z. Kakushadze, A Three-Family SU(6) Type I Compactification, hep-th/9804110; A Three-Family $S U(4)_{c} \otimes S U(2)_{w} \otimes U(1)$ Type I Vacuum, hep-th/9806044;

G. Zwart, Four-dimensional $N=1 \mathbb{Z}_{N} \times \mathbb{Z}_{M}$ Orientifolds, hep-th/9708040;

G. Aldazabal, A. Font, L.E. Ibáñez and G. Violero, $D=4, N=1$, Type IIB Orientifolds, hep-th/9804026

[5] J. Polchinski and E. Witten, Evidence for Heterotic-typeI String Duality, Nucl. Phys. B460 (1996) 525, hep-th/9510169

[6] Z. Kakushadze, G. Shiu and S.-H.H. Tye, Type IIB Orientifolds, F-theory, Type I Strings on Orbifolds and Type I-Heterotic Duality, hep-th/9804092;

G. Shiu and S.-H.H. Tye, TeV Scale Superstring and Extra Dimensions, hepth/9805157;

Z. Kakushadze, On Four Dimensional $N=1$ Type I Compactifications, hepth/9806008

[7] D. Gepner, Space-time Supersymmetry in Compactified String Theory and Superconformal Models, Nucl. Phys. B296 (1988) 757

[8] G. Pradisi, A. Sagnotti and Y.S. Stanev, Planar Duality in SU(2) WZW Models, Phys. Lett. B354 (1995) 279, hep-th/9503207; The Open Descendants of Non-Diagonal SU(2) WZW Models, Phys. Lett. B356 (1995) 230, hep-th/9506014; Completeness 
Conditions for Boundary Operators in 2D Conformal Field Theory, Phys. Lett. B381 (1996) 97, hep-th/9603097;

J. Fuchs and C. Schweigert, Branes:From Free Fields to General Backgrounds, hepth/9712257

[9] A. Recknagel and V. Schomerus, D-branes in Gepner Models, hep-th/9712186

[10] J.L. Cardy, Boundary Conditions, Fusion Rules and the Verlinde Formula, Nucl. Phys. B324 (1989) 581

[11] J. Fuchs and C. Schweigert, Classifying Algebras for Boundary Conditions and Traces on Spaces of Conformal Blocks, hep-th/9801191

[12] J. Fuchs and C. Schweigert, A Classifying Algebra for Boundary Conditions, Phys. Lett. B414 (1997) 251, hep-th/9708141

[13] C. Angelantonj, M. Bianchi, G. Pradisi, A. Sagnotti and Ya.S. Stanev, Comments on Gepner Models and Type I Vacua in String Theory, Phys. Lett. B387 (1996) 743, hep-th/9607229

[14] J. Fuchs, A.N. Schellekens and C. Schweigert, A Matrix S for all Simple Current Extensions, Nucl. Phys. B473 (1996) 323, hep-th/960078

[15] M. Bianchi, G. Pradisi and A. Sagnotti, Toroidal Compactifications and Symmetry Breaking in Open String Theories, Nucl. Phys. B376 (1992) 365;

Z. Kakushadze, G. Shiu and S.-H.H. Tye, Type IIB Orientifolds with NS-NS Antisymmetric Tensor Backgrounds, hep-th/9803141

[16] R. Blumenhagen, R. Schimmrigk and A. Wißkirchen, $(0,2)$ mirror symmetry, Nucl. Phys. B486 (1997) 598, hep-th/9609167;

R. Blumenhagen and S. Sethi, On orbifolds of $(0,2)$ models, Nucl. Phys. B491 (1997) 263, hep-th/9611172;

R. Blumenhagen and M. Flohr, Aspects of $(0,2)$ orbifolds and mirror symmetry, Phys. Lett. B404 (1997) 41, hep-th/9702199;

R. Blumenhagen, Target Space Duality for $(0,2)$ compactifications Nucl. Phys. B513 (1998) 573, hep-th/9707198; (0, 2) Target Space Duality, CICYs and Reflexive Sheaves, Nucl. Phys. B514 (1998) 688, hep-th/9710021;

A. Sen and S. Sethi, the Mirror Transform of typeI Vacua in Six Dimensions, Nucl. Phys. B499 (1997) 573, hep-th/9703157 